\title{
Blog Educativo BioquímicAtiva: Uma Forma de Vivenciar a Educação em Bioquímica e sua Relação com a Fisiopatologia
}

\author{
Fernando Miguel Pedroso Toyos, Ana Karina Furtado Mendes e Tatienne Neder Figueira \\ da Costa*
}

Curso de Graduação em Nutrição da Universidade Federal do Tocantins

* Autora para correspondência: tatienneneder@uft.edu.br

\section{RESUMO}

O projeto de extensão BioquímicAtiva surgiu com o intuito de utilizar a internet como um meio de ensino da Bioquímica, dada a dificuldade que os alunos comumente relatam sobre a compreensão dessa disciplina e sua associação com a prática profissional. Por essa razão, o presente trabalho avaliou a eficiência de um $b l o g$ educativo sobre Bioquímica, elaborado a partir de um post de fácil linguagem, didático e interativo, utilizando-se da interdisciplinaridade com a Fisiopatologia. Toda a criação do blog e de seu conteúdo foi executada pelos integrantes do projeto, os quais se basearam na literatura científica para a criação do post "Você Sabe o Que É Esteatose Hepática Alcoólica?". Para a avaliação do blog consideraram-se parâmetros de visão geral e informações demográficas, gerados pelo Google Analytics. O conteúdo postado foi avaliado pela enquete "O Que Você Achou Deste Conteúdo?” - disponível ao final do post para votação. O blog foi avaliado ao longo de quinze dias, contados a partir da data de sua divulgação; com registro total de 1236 visualizações nesse período, incluindo usuários de diversas cidades do Brasil e de outros países. Foram notificados 26 votos, sendo 25 para a opção "Excelente" (96,15\%), um para "Bom" (3,84\%) e nenhum voto para "Indiferente". Avalia-se que o projeto elaborado foi positivo, o que demonstra a importância do uso de novos métodos de ensino e tecnologias que possam contribuir para a formação e o interesse pela Bioquímica, bem como estimular seu uso para outros cursos de graduação, a fim de melhorar o processo de ensino e aprendizagem dos acadêmicos.

Palavras-chave: Blog Educacional; Disciplina de Bioquímica; Interdisciplinaridade; Nutrição.

\begin{abstract}
The project of extension BiochemicalActive came with the intention of using the internet as a means of teaching Biochemistry, given the difficulty that students commonly report about the comprehension of this discipline and its association with practice. For this reason, the present study evaluated the effectiveness of an educational blog about Biochemistry, elaborated from a post of easy language, didactic and interactive using the interdisciplinarity with the Pathophysiology. All the creation of the blog and its content was executed by the members of the project, who based on the scientific literature to create the post "Do You Know What Is Alcoholic Liver Steatosis?". For the evaluation of the blog it was considered parameters of overview and demographic information, generated by Google Analytics. The content posted was evaluated by the poll "What Did You Think Of This Content?" available at the end of the post for voting. The blog was evaluated for 15 days, counted from the date of its disclosure, with a total of 1236 views in this period, including users from several Brazilian cities and some countries. "Good" $(3.84 \%)$ and no votes to be indifferent. Our evaluation is that the project was positive, which demonstrates the importance of using new teaching methods and technologies that can contribute to the formation and interest in Biochemistry, as well as stimulate its use for other undergraduate courses, thus improving the process of teaching and learning of academics.
\end{abstract}

Keywords: Educational Blog; Discipline of Biochemistry; Interdisciplinarity; Nutrition.

\section{Introdução}

A procura pelo curso de Nutrição como área de formação profissional, bem como o número de cursos de Graduação em Nutrição no Brasil têm crescido substancialmente ao longo dos anos. Desde sua criação, em 1939, até 1969, foram instituí- 
dos apenas mais seis cursos no Brasil. A partir da década de 1970, começou um processo de expansão do número de cursos no país (RECINE et al., 2012). Dados do sistema e-Mec demonstram que entre 1998 e 2002 foram criados 110 novos cursos de Nutrição em diversos estados brasileiros e atualmente tem-se o registro de 531 cursos em todo o país (e-MEG, 2016).

Considerando-se a atual conjuntura epidemiológica e nutricional brasileira, na qual a desnutrição, o excesso de peso e suas comorbidades fazem parte de um cenário problemático de saúde pública, a formação do nutricionista vem se tornando cada vez mais complexa, exigindo do mesmo maior integralidade entre os variados conteúdos abordados durante a graduação (RECINE et al., 2012).

Segundo estudo realizado por Bianco (2007), 100\% dos 82 cursos de Nutrição do Estado de São Paulo existentes nesse ano possuíam a disciplina de Bioquímica em sua matriz curricular, dado que demonstra a relevante aplicabilidade desse conhecimento nos mais diversos campos de atuação profissional (ALBUQUERQUE et al., 2012) como Fisiopatologia, Nutrição Esportiva e Alimentação.

Sabe-se que para a compreensão da fisiopatologia de uma doença é necessário utilizar-se dos conhecimentos bioquímicos. Dessa forma, o estudo conjunto de tais saberes é essencial para o melhor entendimento das diversas patologias e seus respectivos aspectos clínicos - como interpretação de exames laboratoriais. Nesse contexto, o processo educativo deve ter por objetivo informar e problematizar as questões do cotidiano, de forma a criar uma reflexão por parte do aluno que cause impacto e remeta a discussões mais profundas (BOOG et al., 2003).

De modo similar, a Nutrição Esportiva, considerada uma das sete áreas de atuação do nutricionista (CFN, 2005), também requer uma base bioquímica para seu entendimento, levando-se em conta que o exercício físico induz adaptações metabólicas, que deverão impactar diretamente nas estratégias nutricionais a serem traçadas. Em adição, correlacionar a Bioquímica à Nutrição Esportiva faz despertar no aluno o senso crítico sobre inúmeras estratégias e suplementos nutricionais que surgem a cada dia.
Ainda que relevante e apresentada de forma organizada nos currículos tradicionais, a Bioquímica comumente é definida pelos alunos como uma coleção de estruturas químicas e reações, dificilmente assimiladas e desintegradas de sua prática profissional. Isso gera grande desinteresse pela disciplina, como verificado pelo estudo de Bianco (2007). Ao avaliar a percepção dos alunos do curso de Nutrição da USP em relação à disciplina de Bioquímica, aquele autor observou que em diversos momentos os discentes pontuaram a importância de relacionar a Bioquímica à Nutrição Esportiva, bem como de maior aproximação desse conteúdo com a Nutrição; além do precário entendimento dos conteúdos abordados e da falta de associação entre a disciplina e a atividade profissional (BIANCO, op. cit.).

Esse cenário aponta para a emergente modificação do ensinar bioquímico nos cursos da área de saúde, utilizando-se de meios e métodos de ensino, como a internet, que possibilitem despertar maior motivação e interesse dos alunos pelo conteúdo e que facilitem o processo de ensino-aprendizagem, não apenas a memorização de informações.

O uso da informática e da internet tem se tornado uma ferramenta cada vez mais frequente para produzir conhecimento, dada sua facilidade de acesso e flexibilidade. Utilizar tal meio de comunicação para ampliar os métodos de ensino pode ser útil na tentativa de inserir e engajar o aluno dentro do ambiente acadêmico, melhorando seu aprendizado e retenção de informação (LOBO, 2015). Por essa razão, o objetivo do presente trabalho foi avaliar a eficiência de um blog educativo com abordagem bioquímica a partir de um texto (post) de fácil linguagem, didático e interativo, utilizando-se da interdisciplinaridade com conteúdos de Fisiopatologia como proposta de melhorar o processo de ensino-aprendizagem.

\section{Desenvolvimento}

\section{Descrição do Estudo}

O presente estudo é resultado de um projeto de extensão cadastrado no Sistema de Informação e Gestão de Projetos (SIGProj), que foi desenvolvido 
por dois acadêmicos do curso de Graduação em Nutrição da Universidade Federal do Tocantins (UFT), sob coordenação de uma professora desse curso, responsável pelas disciplinas de Processos Bioquímicos Aplicados à Nutrição e Fisiopatologia da Nutrição na referida instituição. $O$ fruto de tal ação extensionista culminou no desenvolvimento e na criação de um blog educativo intitulado BioquímicAtiva.

\section{Desenvolvimento e Criação do Blog}

Para a criação do blog educativo foi aberta uma conta no Gmail com o nome BioquímicAtiva e posteriormente outra no Wix (https://www.wix.com), uma plataforma gratuita que auxilia internautas na criação de sites, com disponibilidade de layouts predefinidos e opções de design e configurações de páginas. O layout que melhor atendeu a presente proposta foi o de blog, e em seguida foram feitas as alterações para sua personalização.

O acesso ao blog permite adentrar as opções "Sobre" e "Posts". A primeira destina-se a uma breve contextualização sobre o estado da arte do assunto proposto (ensino interdisciplinar e aplicável da Bioquímica) e direciona o leitor para o objetivo do presente estudo. Na página de posts, além da identificação dos membros do projeto apresentada no item "Quem Somos", ficaram expostos os posts intitulados "Bem-vindos ao Site", "Esteatose Hepática Alcoólica", "Consumo de Frutose" e "Esteatose Hepática", com visualização parcial de seus conteúdos e acesso completo em outra sessão do blog após um clique sobre a imagem disponível ou sobre a opção "Ler Mais".

Para a personalização dos posts foram utilizados: recursos de imagem; opções de fonte, formatação e cores; imagens e gifs.

A página sem um domínio pago leva o nome wix em seu endereço eletrônico, além de propagandas da plataforma. Como o intuito foi desenvolver um site independente, o domínio foi feito pelo site registro.br, após verificação de disponibilidade do domínio BioquímicAtiva, sendo em seguida ativado. A hospedagem anual foi feita pelo site Wix e entre os planos Premium disponíveis selecionou-se a modalidade Combo com 3 GB de armazenamento, 2 GB de largura de banda e remoção de anúncios do Wix. Dessa forma, o endereço eletrônico do site criado foi: www.bioquimicativa.com.br.

O site desenvolvido também foi cadastrado pelo URL (do inglês, Uniform Resource Locator), através de uma conta no Google Analytics (serviço oferecido pelo Google), o qual foi utilizado como instrumento de análise e geração dos dados estatísticos, assim como foi elaborada uma enquete ao final do post sobre esteatose hepática alcoólica.

\section{Elaboração do Material Educativo}

Inicialmente foram selecionados três grandes campos do conhecimento os quais a Bioquímica permeia, a saber: Nutrição Esportiva, Fisiopatologia e suas repercussões clínicas, e Alimentos, de maneira geral, as quais subsidiaram a escolha dos textos para a elaboração dos materiais educativos, com vistas à interdisciplinaridade.

Contudo, para o presente estudo, a temática desenvolvida baseou-se na Fisiopatologia e suas repercussões clínicas. Especificamente na esteatose hepática alcoólica, com ênfase nos aspectos bioquímicos desse agravo, de forma a possibilitar o entendimento da referida patologia.

O material foi produzido em forma de texto, alternado com imagens, diálogo interativo, figuras e gifs, com o intuito de propiciar uma linguagem mais simples, dinâmica e atrativa para um assunto tido como complexo e, dessa forma, motivar a leitura e facilitar o aprendizado, sem deixar de garantir a abordagem do conteúdo de maneira profunda e baseada em evidências científicas. Para tal, a bibliografia consultada se deu por meio de artigos científicos retirados do banco de dados Pubmed e livros de Bioquímica, contidos ao final do post de esteatose hepática alcoólica.

\section{Divulgação do Blog}

Os meios de divulgação do blog se deram através de rede social, a partir da criação de uma conta no Facebook, bem como pela divulgação do endereço eletrônico do site BioquímicAtiva via e-mail para as coordenações e turmas dos cursos da área da saúde 
da UFT e Centros Acadêmicos, além de e-mail para professores de outras Instituições de Ensino Superior. O blog foi publicado em domínio próprio no dia 14 de novembro do ano de 2016, sendo essa também a data de sua divulgação.

\section{Avaliação do Blog}

Para a avaliação do blog consideraram-se os resultados obtidos por um período de quinze dias, contados a partir da data de sua divulgação. De acordo com os relatórios gerados pelo Google Analytics, foi possível avaliar parâmetros sobre visão geral, os quais compreenderam: sessões (período de tempo em que o utilizador está interagindo ativamente com o website), total de usuários (utilizadores que interagiram com a página, incluindo os novos e os que retornaram), visualizações de página (número total de páginas visualizadas, sendo contabilizadas também as visualizações repetidas de uma única página), número médio de páginas visualizadas durante uma sessão, taxa de rejeição (visitas de página única, ou seja, a pessoa abandonou o site de entrada sem ter interagido com a mesma) e também porcentagem de novas sessões. Por meio dessa ferramenta utilizada, foi possível determinar também as informações demográficas, como os países e cidades que acessaram $o b l o g$.

Para a avaliação da eficiência do material educativo elaborado, foi criada uma enquete disponível ao final do post sobre esteatose hepática alcoólica, a qual continha a seguinte pergunta: "O Que Você Achou Deste Conteúdo?”. A partir dessa pergunta, o usuário tinha a opção de selecionar uma dentre três respostas: "Excelente", "Bom" ou "Indiferente".

Os resultados obtidos foram expressos de forma absoluta ou relativa (\%), a depender do dado considerado.

\section{Resultados}

Ao longo de quinze dias, houve um total de 1236 visualizações das páginas, incluindo visualizações repetidas de uma única página. Os demais parâmetros de visão geral encontram-se na Tabela 1 . A taxa de rejeição foi de $40,04 \%$ e a porcentagem de novas sessões chegou a $60,71 \%$. O pico mais alto de sessões ocorreu no dia 14 de novembro, data em que o site entrou no ar.

\begin{tabular}{|l|l|}
\hline VISÃO GERAL & $\mathbf{n}$ \\
\hline Total de sessões & 537 \\
\hline Novos usuários & 326 \\
\hline Retornos de visitas & 211 \\
\hline Páginas por sessão & 2,3 \\
\hline
\end{tabular}

Tabela 1 - Parâmetros de visualização geral do site, no período de 14 a 29 de novembro de 2016. Fonte: Relatório do Google Analytics.

\begin{tabular}{|l|l|l|}
\hline PAís & $\mathbf{n}$ & Percentual (\%) \\
\hline BRASIL & 441 & 82,2 \\
\hline RÚSSIA & 91 & 16,95 \\
\hline AUSTRÁLIA & 1 & 0,19 \\
\hline SUÉGIA & 1 & 0,19 \\
\hline NÃO IDENTIFICADO* & 3 & 0,56 \\
\hline TOTAL & 537 & 100 \\
\hline
\end{tabular}

Tabela 2 - Número de acessos realizados no site, segundo país de ocorrência no período de 14 a 29 de novembro de 2016. Fonte: Relatório do Google Analytics. Nota: * Refere-se ao dado que o Google Analytics não consegue identificar. 
Especificamente no território brasileiro, foram identificados acessos em cinco estados e DF, com as respectivas cidades representadas na Tabela 3. Pôde-se notar que a cidade com maior número de acessos foi Palmas.

A enquete "O Que Você Achou Deste Conteúdo?", ao final do texto postado com o título "Você Sabe o Que É Esteatose Hepática Alcoólica?”, obteve um total de 26 votos, dentre os quais 25 foram para a opção "Excelente" (96,15\%), um para "Bom" (3,84\%) e nenhum voto para "Indiferente".

\section{Discussão}

Um dos principais achados de nosso estudo foi que, apesar do curto período de tempo destinado à avaliação, obtivemos um elevado número de visualizações do blog e o acesso do conteúdo realizado por usuários de diferentes cidades, tanto nacionais (representadas por cinco estados brasileiros e DF) como internacionais. Isso demonstra a importância do uso da internet como uma ferramenta de fácil e ampla acessibilidade, capaz de atingir diversos públicos com interesses em comum.

Dentre os inúmeros recursos da internet, destacam-se os blogs, instrumento educativo escolhido para o presente trabalho e que vem sendo cada vez mais empregado nos diferentes campos do conhecimento (BARRO et al., 2016; JUNIOR et al., 2015). Estruturalmente, os blogs apresentam-se na forma de uma página web, contendo pequenos blocos de textos apresentados de modo cronológico inverso, ou seja, com o conteúdo mais recente sendo disposto no topo da página. Esses blocos de textos são denominados posts e são classificados em categorias de acordo com o assunto (BARRO et al., 2016).

A utilização de blogs como um recurso tecnológico e estratégico de ensino contribui para o aprendizado dos estudantes, uma vez que se torna flexível pela sua disponibilidade e facilidade de acesso, aproximando os leitores de assuntos do seu interesse de forma dinâmica (MARQUES \& ABEGG, 2012).

Nesse sentido, a utilização de novas formas de ensino, especialmente envolvendo conteúdos de alta complexidade, como a Bioquímica, vem sendo proposta com o intuito de melhorar o processo de ensino e aprendizagem (LOBO, 2015; ALBUQUERQUE et al., 2012). Por ser uma disciplina comumente trabalhada de maneira isolada e fragmentada, muitos alunos a consideram como desvinculada da realidade profissional (BIANCO, 2007).

\begin{tabular}{|l|l|l|}
\hline CIDADE & $\mathbf{n}$ & Percentual (\%) \\
\hline PALMAS & 275 & 51,21 \\
\hline GOIÂNIA & 23 & 4,28 \\
\hline SÃO PAULO & 15 & 2,79 \\
\hline BELO HORIZONTE & 14 & 2,61 \\
\hline GAMPINAS & 13 & 2,42 \\
\hline RIO DE JANEIRO & 7 & 1,30 \\
\hline BRASÍLIA & 6 & 1,12 \\
\hline SAMARA & 28 & 5,51 \\
\hline SAINT PETERSBURG & 60 & 11,17 \\
\hline NÃO IDENTIFICADO* & 10 & 1,86 \\
\hline TOTAL & 537 & 100 \\
\hline
\end{tabular}

Tabela 3 - Número de acessos realizados no site, segundo cidade de ocorrência, no período de 14 a 29 de Novembro de 2016. Fonte: Relatório do Google Analytics. Nota: * Refere-se ao dado que o Google Analytics não consegue identificar. 
Em decorrência de tal motivo, esses não visualizam sua aplicabilidade, preocupam-se apenas em obter aprovação na disciplina e, por conseguinte, não sentem interesse por ela, como demonstrado no estudo de Bianco (2007).

Ao avaliarmos o nível de satisfação dos usuários em relação ao conteúdo de esteatose hepática postado, acreditamos ter propiciado aos leitores uma visão mais aplicável e dinâmica da Bioquímica, permitindo, dessa forma, maior aproximação da teoria com sua prática.

Ademais, a participação de graduandos em projetos de extensão que aprimorem a sua capacidade de produção, criatividade e iniciativa é considerada de extrema importância para o desenvolvimento de habilidades que normalmente são pouco trabalhadas durante a graduação e que, por sua vez, estimulam a comunicação e a busca por novos conhecimentos, contribuindo para a formação de profissionais e futuros docentes mais capacitados (DAUN \& GAMBARDELLA, 2016).

\section{Conclusão}

Podemos concluir que o projeto de extensão BioquímicAtiva, embora avaliado por curto período de tempo, foi positivo e demonstra a importância que novas formas de ensino e tecnologias têm tanto para graduandos envolvidos em atividades extensionistas, quanto para a comunidade acadêmica que usufruirá do material didático despertando, dessa forma, maior interesse pela Bioquímica.

Diante disso, acreditamos que a continuidade do projeto possa fomentar cada vez mais o ensino da Bioquímica a acadêmicos de diversas áreas de atuação ligadas à saúde e estimular o uso de novas tecnologias como métodos de ensino em outros cursos, melhorando assim o processo de ensino e aprendizagem dos acadêmicos.

\section{Referências Bibliográficas}

ALBUQUERQUE, Manuela Alves Cavalcanti; AMORIM, A. H. C.; ROCHA, J. R. C. F.; SILVEIRA, L. M. F. G.; NERI, D. F. M. "Bioquímica como Sinônimo de Ensino, Pesquisa e Extensão: um Relato de Experiência". Revista Brasileira de Educação Médica, vol. 36, n. 1, 2012, pp. 137-142.

BARRO, Mario Roberto; VERAS, Lea \& QUEIROZ, Salete Linhares. "Blogs no Ensino de Química: Análise de Comentários Publicados em Disciplina de Comunicação Científica". Quím. Nova, vol. 39, n. 2, 2016, pp. 238-244.

BIANCO, André Amaral Gonçalves. A Disciplina de Bioquímica para o Curso de Nutrição: uma Proposta de Desenvolvimento de Currículo. Tese (Doutorado em Ciências, Bioquímica), Instituto de Química, Universidade de São Paulo, 2007.

BOOG, Maria Cristina Faber; VIEIRA, C. M.; OLIVEIRA, N. L.; FONSECA, O. \& LABBATE, S. "Utilização de Vídeo como Estratégia de Educação Nutricional para Adolescentes: Comer... o Fruto ou o Produto?". Revista de Nutrição, Campinas, vol. 16, n. 3, 2003, pp. 281-293.

BRASIL. Sistema do Ministério da Educaşão $(M E C)$. Disponível em: <http://emec.mec.gov.br>. Acessado em 12 nov. 2016.

CONSELHO FEDERAL DE NUTRICIONISTAS. "Dispõe sobre a Definição das Áreas de Atuação do Nutricionista e Suas Atribuições, Estabelece Parâmetros Numéricos de Referência, por Área de Atuação, e Dá Outras Providências. Resolução n. 380 de 28 de dezembro de 2005". Brasília, DF, 2005.

DAUN, Felipe \& GAMBARDELLA, Ana Maria Dianezi. "Extensão Universitária na Graduação em Nutrição: Experiências de Produção de Vídeos Educativos". Revista Grad. USP, vol. 1, n. 1, jul. 2016.

JUNIOR, Gilmario dos Santos Freitas; ROCHA, Mario Ferreira da \& ALBUQUERQUE, Monck Charles Nunes de. "Uso do Blog como Ferramenta nos Processos de Ensino e Aprendizagem: Relato de Experiência Vivida em Horizonte-CE". Revista Tecnologias na Educação, vol. 12, julho 2015.

LOBO, Luiz Carlos. "Educação Médica nos Tempos Modernos". Revista Brasileira de Educaşão Médica, vol. 39, n. 2, 2015, pp. 328-332.

MARQUES, Eliandra Gomes \& ABEGG, I. "Blog como Ferramenta Pedagógica na Produção Colaborativa em Educação Ambiental". Jornal de Monografias Ambientais, REMOA/UFSM., vol.10, n. 10, out./dez. 2012, pp. 2115-2127.

RECINE, Elisabetta; GOMES, R. C. F.; FAGUNDES, A. A.; PINHEIRO, A. R. O.; TEIXEIRA, B. A.; SOUSA, J. S.; TORAL, N. \& MONTEIRO, R. A. "A Formação em Saúde Pública nos Cursos de Graduação de Nutrição no Brasil". Revista de Nutrição, vol. 25, n. 1, 2012, pp. 21-33. 